\title{
Glycodeline-A, sFlt-1, PIGF Combined as Predictor Markers of First Trimester Pregnancy Loss in Iraqi Women
}

\author{
Mohammed H. Dawood ${ }^{1}$, Halla G. Mahmood ${ }^{2}$, Afraa M. Nedawi ${ }^{3}$ \\ ${ }^{1}$ Clinical Biochemistry, Ministry of Health, Baghdad, Iraq, ${ }^{2}$ Clinical Biochemisrty, Biochemistry school, Scientific \\ School, University of Baghdad, Baghdad, Iraq, ${ }^{3}$ Clinical Pathology, Department of Pathology, College of \\ Medicine, University of Baghdad, Baghdad, Iraq
}

\begin{abstract}
Objective: evaluate the serum level of Glycodeline-A, sFlt-1, PIGF combined with $\beta$ hCGas predictor markers of first trimester pregnancy loss in Iraqi women .

Method: A case-contol study, carried out from April 2019 to March 2020 and included 85 women, 40 of them as patients which have abortion at the first trimester and 45 of them as healthy control which haven, $\mathrm{t}$ any complication at the same time of gestation, investigations included serum measurements of $\beta \mathrm{hCG}$, Glycodeline-A, sFlt-1, PIGF By Method ELISA.

Results: Serum Niveaus $\beta$ hCG, sFlt-1 Was major higher in healthy controls than in patients, while serum level of Glycodeline-A, PlGF was significantly lower in healthy control than in patients, and there is no significant correlation among each of age with other parameters, and there is no significant correlation among each of gest.age with Glycodeline-A and sFlt-1, while there is significant correlation among each of ges.age with $\beta$ hCG and $\mathrm{PlGF} \mathrm{P}<0.05$.

Conclusion: The present study suggested the significant role of Glycodeline-A, sFlt-1, PlGF in pathogenesis of first trimester pregnancy loss and some of them was related to $\beta$ hCGin this period and the age of patients wasn' $t$ effect on all of these parameters.
\end{abstract}

Keywords: $\beta h C G$, Glycodeline-A, sFlt-1, PlGF.

\section{Introduction}

Pregnancy defined as the condition of carrying fetus at the pregnant woman, and can be confirmed by positive urine or blood test with ultrasound that is detection of fetal heartbeat, an X-ray may be using, this condition stay about 9 months begining from the first day of the (LMP) of the woman, this state is divided ordinarily In three parts, each part DreiMonate period ${ }^{1}$, More important basic cell differentiation Occurrence of fetal during the first three months of pregnancy, so any complication During this stage the embryo is leading to result represent Grave or pregnancy complications, the consistence of blood flow of fetal placental and uteroplacental is the important side of processes of pregnancy and fetal consistence $^{2}$, successful morphogenesis of uterine placental vascular with successful Morphogenicity of fetus blood circulation are the main factor In these
Two Phases systems existing represent these processes of morphogenesis vascular: vasculogenesis: firstly development formation of the blood flow from the activated cells of mesodermal Angiogenesis and : novel vessels Through Blood is formed from present vascular form, which is represent the consistence and development of the system of vascular fetus and placenta through pregnancy. There is no prevent work to protect the pregnant from the miscarriage especially when this situation is occurring whith unknown reason which is undetect, in other site there are many causes that increase the danger of miscarriage, if the pregnant is having difficult maintaining of her carry ${ }^{3}$, the specialist may evaluate some known reasons of miscarriage and remove it through carry, supplemet of hormones and nutrients can help to developing embryo, this growing of embryo happened mostly in the first three months of carry, in 
most cases miscarriages due to the defect of normally fetus growing ${ }^{4}$, the various symptoms of miscarriage depend on the time of carry age, it happens rapidly even before she know pregnant, these signs include bleeding vagina $^{5}$, tissue drainage,cramping or severe lower pain,back pain mild to heavy, Growth factors play a vital role in the development of vessels; they function as cell mitogens; as attractants in the creation of vascular architectonics; and most notably, as morphogens; the major regulators of angiogenesis are part of the $\mathrm{VEGF}^{6}$ complex. In addition to direct angiogenesis activators, there is a wide number of causes, including matrix metalloproteinases (MMPs) and their tissue inhibitors (TIMPs), whose effect on angiogenesis is non-specific. production of antiangiogenic Factors are an important part of natural angiogenesis, leading to molecular communication. vascularization ${ }^{7}$, The manufacture of inhibitors acts as a deterrent to unnecessary invasion of trophoblasts, as well as a deterrent to the further growth of the vascular bed and vascularization of pathologically affected tissue locations, angiogenic factors expressed primarily in endothelium and placenta during pregnancy, including receptors VEGFR1 (sFlt1), VEGFR2 (Flk1, KDR) and VEGFR3 (Flt4) ${ }^{8}$, soluble variants of these receptors are able to bind growth factors in circulation, halting or blocking angiogenesis, Humoral factors implicated in vascular development processes are more available for study in maternal circulation, and alteration in their content in mother's blood indicates shifts in the quality of these factors in the fetal blood circulation and tissues9. In this regard, a detailed analysis of variables and their ratios correlated with angiogenesis is critical for understanding and predicting vascular morphogenesis disorders during pregnancy ${ }^{10}$,Apparently Soluble tyrosine kinase-1-like fms (sFlt-1) interferes with the events that Inhibiting central angiogenesis and/or blocking invasion of trophoblast. Improved development of Fms-like receptor of tyrosine kinase -1 (sFlt-1) soluble antiangiogenic agent The placenta contributes to the obstetric Physiotherapy . sFlt-1 Is expressed at very high trophoblast rates, and its development in hypoxic conditions is greatly increased role sFlt- 1 in pregnancy failures is the focus of ongoing investigation ${ }^{11}$. SFlt-1 in maternal circulation therefore decreased was recently Proposed as a probable distinguishing feature the peril of defeat of pregnancy,PlGF is the angiogenic component most commonly controlled in an uncomplicated first trimesterdeciduas, Discovering the involvement of angiogenic factors (sFlt-1/PlGF) in the underlying placental disorder pathophysiology, taking into consideration thethe biomarkers correlated with angiogenesis are related to a High placental insufficiency disorder, these markers are Relevant to early detection and forecasting assessment ${ }^{12}$, Glycodelin-A ( $\left.\mathrm{GdA}\right)$ is an abundant first- trimester glycoprotein deciduas ${ }^{13}$. It participates in fetomaternal defense and early placental growth of different immune cells via its regulatory activities, glycodelin-A is a glycoprotein Consistent with its average expression between 6 and 12 weeks of development in the decidua,Abnormal endometrial rates of glycodelin-A, uterine flushes, and/or maternal serum associate with unexplained miscarriage, early termination of child, and recurrence miscarriage ${ }^{14}$.

Aim of the study: To study its position Glycodeline-A, sFlt-1, PlGFcombined as predictor markers of first trimester pregnancy loss in Iraqi women.

\section{Method}

Study Design: The present project was conducted at the branch of Biochemistry, Medicine College, Baghdad University, at Hospital of Baghdad Teaching through the time from April 19 to March 2020, it included 85 pregnant woman identified the first day of LMP who are confirmed positive pregnancy test in blood, and established to have had pregnancy by Consultant Gynaecologist and matching age 21-35 year and same time 8-12 week of pregnancy and encountered during their attendance the Gynaecology consultation clinic at Baghdad Teaching hospital, encountered these women until the end of week 12 of pregnancy, 40 of them who have pregnancy loss at the prognosis period are patients and the other 45 healty control who completed the same period without problems, a written consent was applied by each patient and an ethical approval had been suitable the researcg based of the Medicine College, Baghdad University and the link with Ministry of Health and enviroment.

Data Setting: Eight to ten milliliters (mls) of blood from subjects are draw out and transferred into plain tube, allows for half hour to sure that is clotting, after this, the specimen must be centrifuged to separated the needed serum at $2500 \mathrm{rpm}$ for limited time roughly ten minuted and stored at freezing degree about $-20 \mathrm{Co}$ up to beginning of the assay the project biochemical markers : Beta HCG, Glycodeline-A, sFlt-1, PIGF By ELISA method

Inference by Statistics: Inference by statistics is a method that analysis of details by using SPSS, this 
information were putting as simple measures of variants (mean, standard deviation and others), qualitative detailes which are significant statistically were using student-t-test for compare between two independent means or ANOVA test for compare between different qualitative detailes on, the statistical significance is equal or less than 0.05 , the coefficient of correlation value $r$ : is a kind of coefficient correlation clarify the connection between two different factors that are calculated on the same period, $r$ may be positive as direct correlation or negative as inverse correlation.

\section{Results}

Explain by table scales of various markers and their correlations according to the study assessment .

Table 1: Results and their obtained correlations for all markers in this study

\begin{tabular}{|l|c|c|c|c|c|}
\hline \multicolumn{7}{|c|}{ GAR00013 } & N & Mean & Std. Deviation & Std. Error Mean \\
\hline Age & 1.00 & 40 & 27.1250 & 4.18292 & .66138 \\
\hline cAge & 2.00 & 45 & 27.2444 & 4.11293 & .61312 \\
\hline Gest Age & 1.00 & 40 & 9.6000 & 1.29694 & .20506 \\
\hline cGestAge & 2.00 & 45 & 9.5333 & 1.09959 & .16392 \\
\hline Beta HCG & 1.00 & 40 & 1024.7740 & 459.39406 & 72.63658 \\
\hline cBeta HCG & 2.00 & 45 & 2309.9409 & 3007.11697 & 448.27453 \\
\hline Glycodein A & 1.00 & 40 & 70.9447 & 56.87327 & 8.99245 \\
\hline cGlycodlin A & 2.00 & 45 & 193.7416 & 200.56648 & 29.89868 \\
\hline sFlt1 & 1.00 & 40 & 118.2153 & 76.42478 & 12.08382 \\
\hline csFlt1 & 2.00 & 45 & 22.2696 & 19.16359 & 2.85674 \\
\hline PIGF & 1.00 & 40 & 141.4350 & 284.10843 & 44.92149 \\
\hline CPIGF & 2.00 & 45 & 340.2947 & 387.01779 & 57.69321 \\
\hline
\end{tabular}

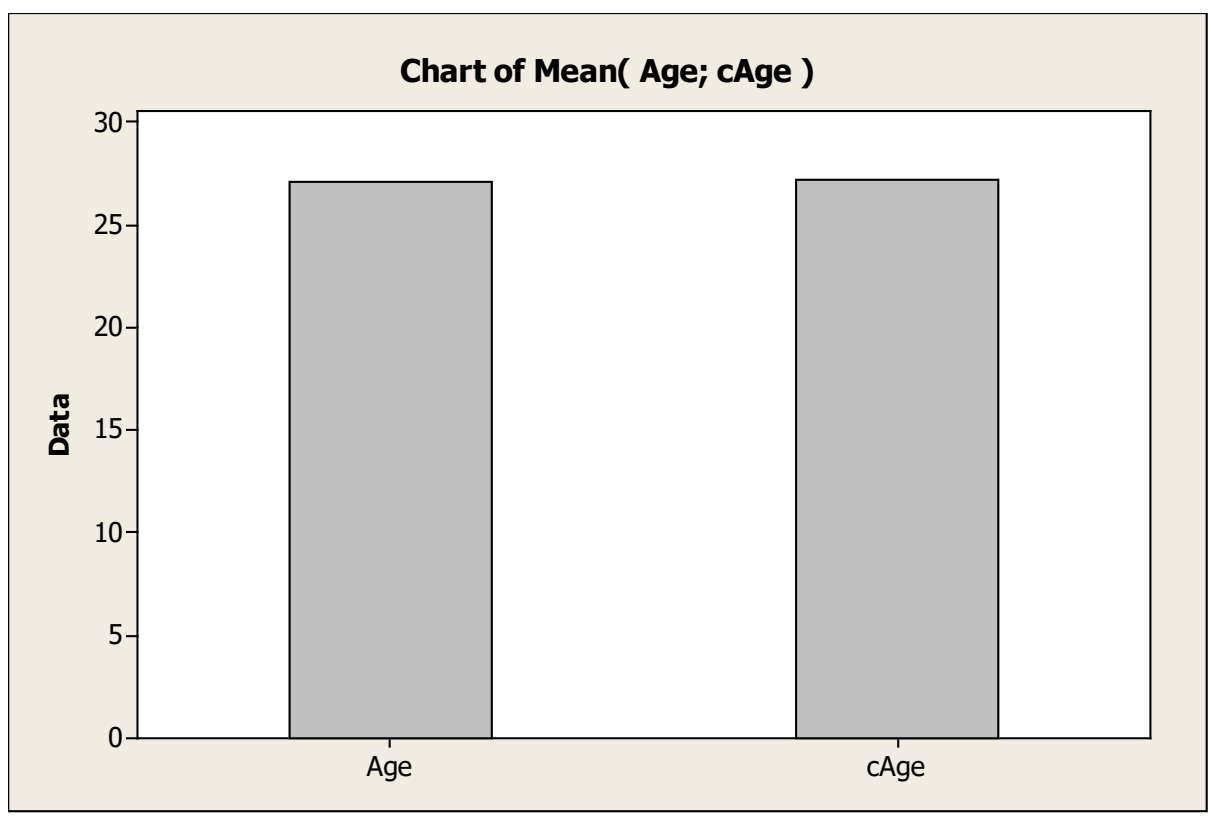

Figure 1: Chart of mean (Age, cAge) 


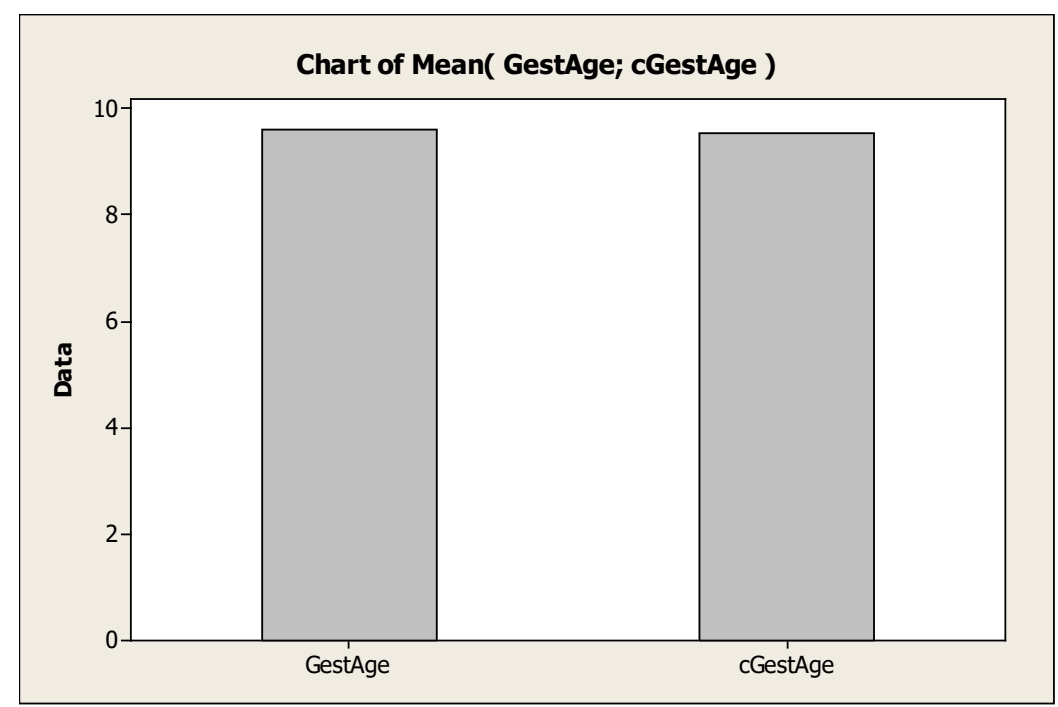

Figure 2: Chart of mean (Gest. age,cGestational age)

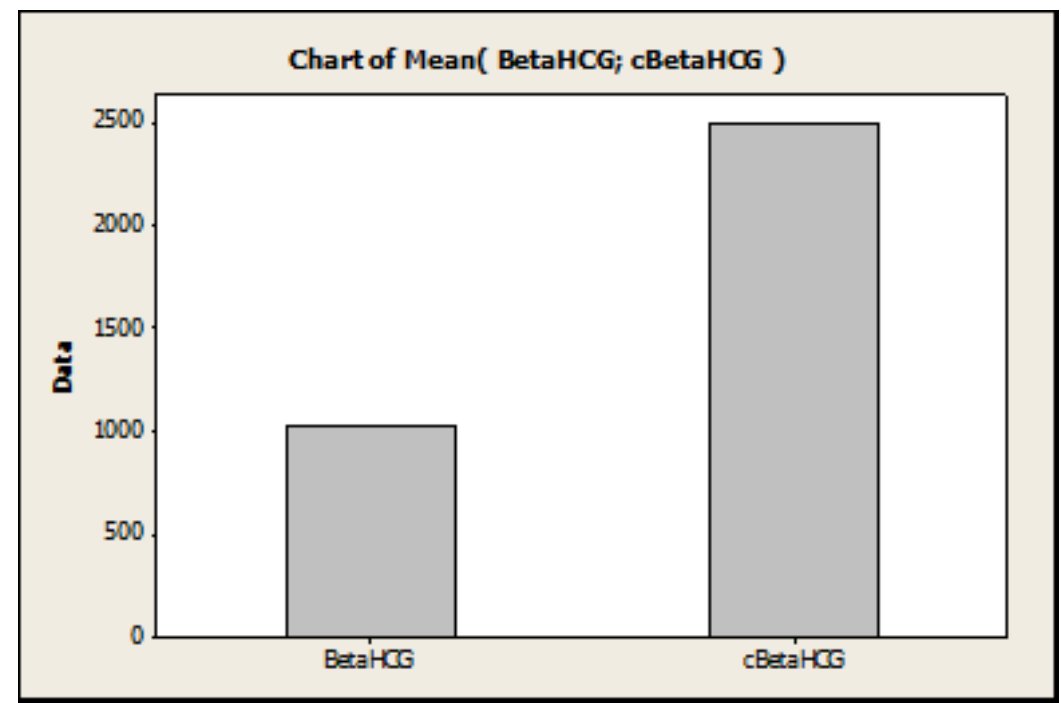

Figure 3: Chart of ( $\beta \mathrm{hCG}, \mathrm{c} \beta \mathrm{hCG})$

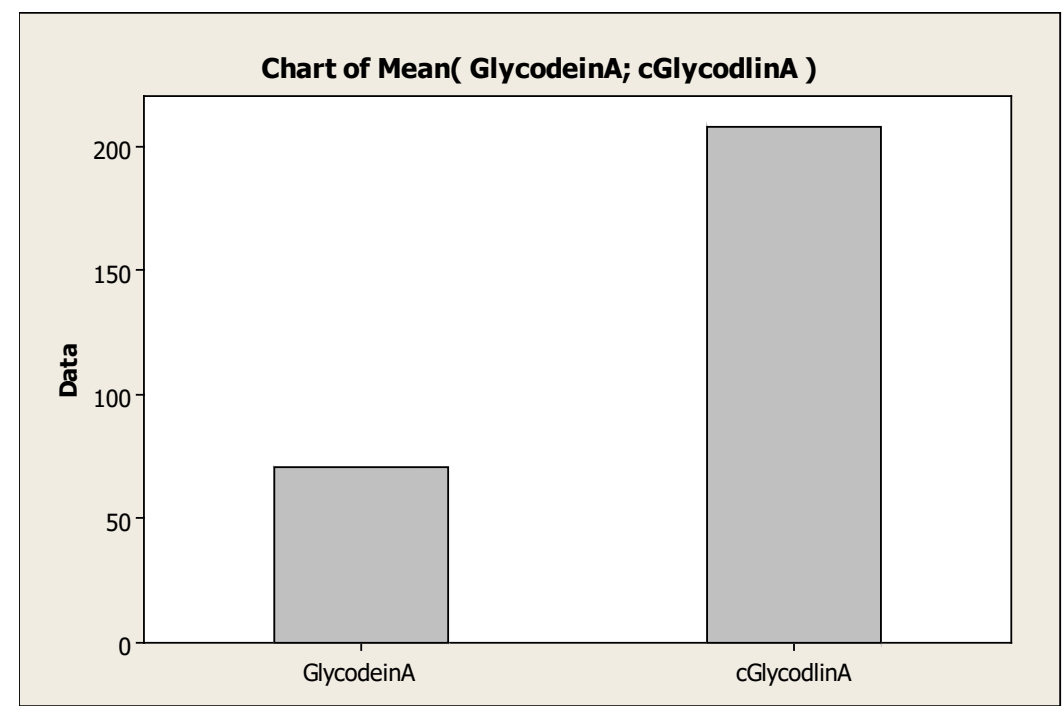

Figure 4: Chart of (Glycodelin-A, cGlycodeline-A) 


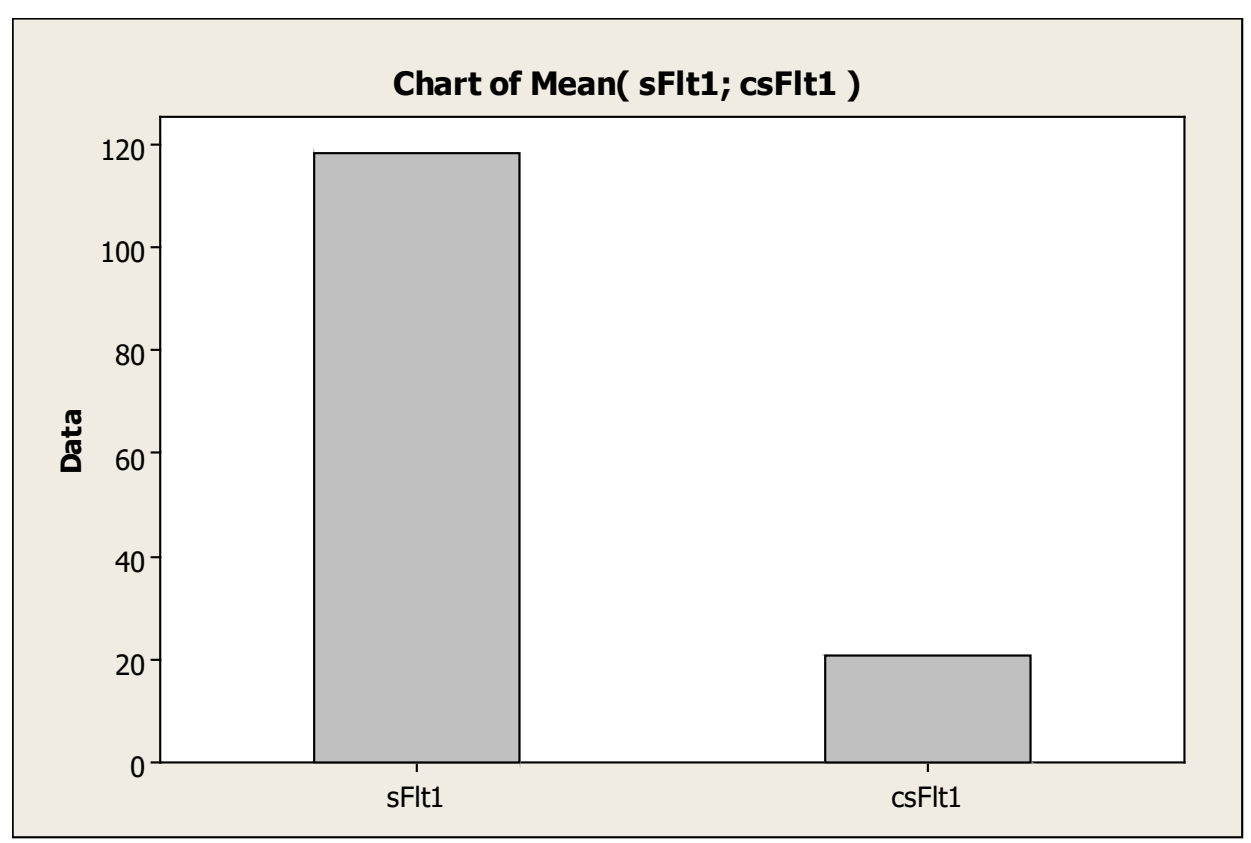

Figure 5: Chart of (sFlt-1, csFlt-1)

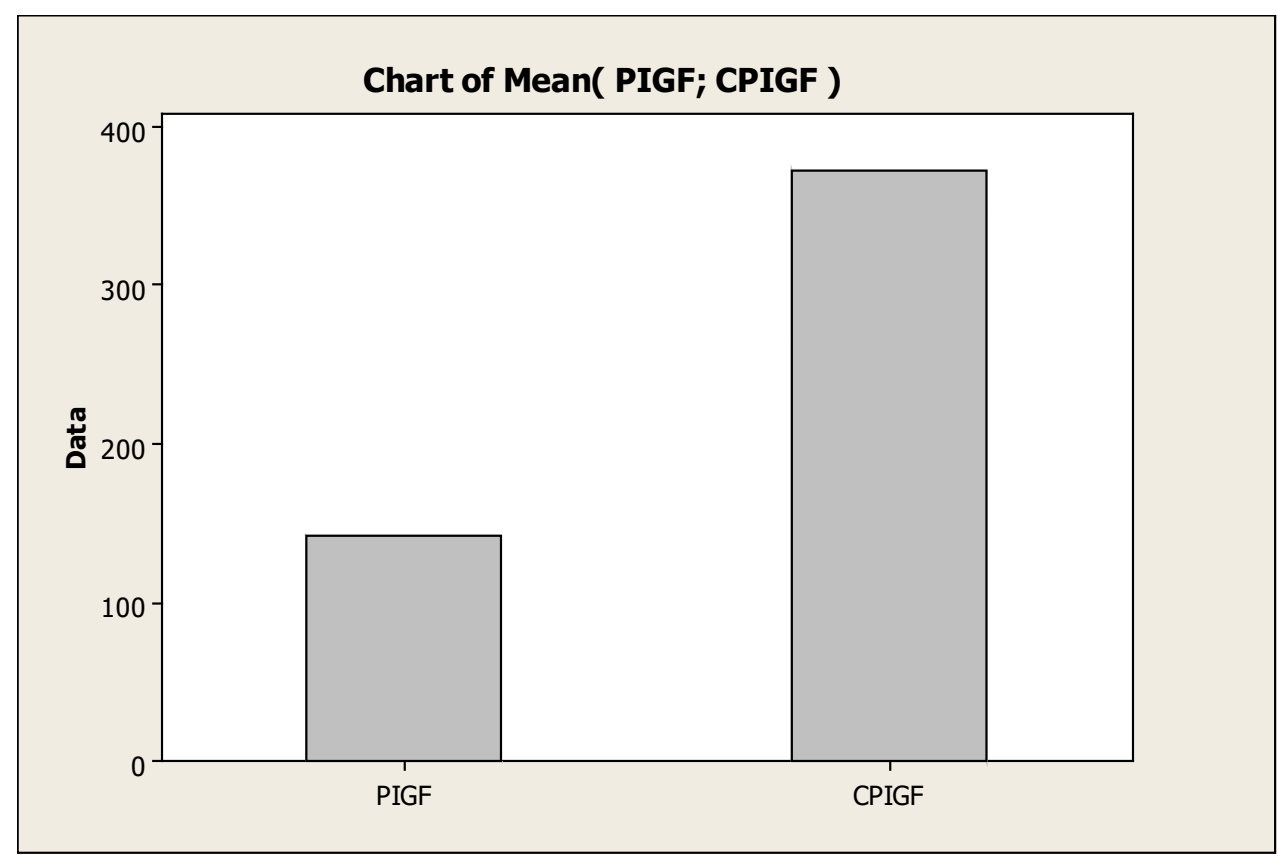

Figure 6: Chart of (PIGF, cPIGF)

\section{Discussion}

Serum level of $\beta \mathrm{h}$ in healthy control group (mean \pm SD $2309.940 \pm 3007.116)$ in patients which have abortion (1024.774 \pm 4 59.394) which show significant correlation : $\mathrm{P}$ value 0.05 , that mean the level of this marker in patients was lower than in healthy control, The present study shows that the serum level of glycodelin-A in healthy control group (mean \pm SD
$193.741 \pm 200.566)$, in patients which have abortion (70.944 \pm 56.873$)$ which show significant correlation : $\mathrm{P}$ value 0.01 , that mean the level of this marker in patients was lower than in healthy control, The present study shows that the serum level of sFlt-1 in healthy control group (mean \pm SD $22.269 \pm 19.163$ ), in patients which have abortion (118.215 \pm 76.424$)$ which show significant correlation: $P$ value 0.05 , that mean the level of this marker in patients was higher than in healthy 
control, The present study shows that the serum level of PlGF in healthy control group (mean \pm SD 340.294 $\pm 387.017)$, in patients which have abortion (141.453 \pm 284.108) which show significant correlation : $P$ value 0.05 , that mean the level of this marker in patients was lower than in healthy control, most of previous projects have found that the chromosomal agents are effective factor in carry loss ${ }^{15}$, old studies have demonstrated that the normal carry depends on normal immune system which is consist of immune defence, immune response, cytokines $^{16}$, the immune effect is important factor in first carry failed, the immune units that establish at the mediater of placenta and the uterus were stay overlap layer of arrangment by immunity system of mother, this process may help of placental growth but some times reduce the placental aggress the embryo, in the time of implantation natural killer units go to the uterus and help the stimulation secrete of cytokines that inhibit the trophoblast aggress ${ }^{17}$.

\section{Conclusion}

Scales of all markers according to the result which obtained from the assessment of them are clarify the relation between patients which have abortion in the first trimester and healty control, these results suggest for $\beta$ hCG: level of this marker in Patients were beyond safe regulation, and for Glycodelin-A: level of this marker in Patients fall beyond safe monitoring,and for sFlt-1: level of this marker in has been careful higher as in healthy check, and for PlGF: level of this marker in patients were beyond safe regulation .

Ethical Approval: Describe written consent was applied by each patient and an ethical approval had been suitable the researcg based of the Biochemistry Department,Medicine College, Baghdad University and the link with Iraqi Ministry of Health .

Funding Source: Making of this work depend on authors only.

\section{Conflict of Interest: No}

Source of Funding: Self funded

Ethical Clearance: Not Required

\section{References}

1. Moran, L. J., McNaughton, S. A., Sui, Z., Cramp, C., Deussen, A. R., Grivell, R. M., \& Dodd, J. M. The characterisation of overweight and obese women who are under reporting energy intake during pregnancy. BMC pregnancy and childbirth.2018, 18(1), 204.

2. Meriwether, K. V., Antosh, D. D., Olivera, C. K., Kim-Fine, S., Balk, E. M., Murphy, M., ... \& Crisp, C. C. Uterine preservation vs hysterectomy in pelvic organ prolapse surgery: a systematic review with meta-analysis and clinical practice guidelines. American journal of obstetrics and gynecology, 2018. 219(2), 129-146.

3. MacGregor, A. Efficacy and side-effects of oral contraceptives. Prescriber,2017. 28(8), 19-26.

4. MacGregor, A. Efficacy and side-effects of oral contraceptives. Prescriber, 2017 28(8), 19-26.

5. Oats R, Abraham V, Llewellyn-Jones, Fundamentals of Obstetrics and Gynaecology. 05 Jan 2016.

6. Hacker,Gambone \& Hobel, Moore, Essentials of Obstetrics and Gynecology.10 Nov 2018.

7. Beischer, MacKay Permezel, Walker, Kyprianou, Obstetrics and Gynaecology and the Newborn .11 Sept 2015.

8. Magowan, Owen \& Thomson, Clinical Obstetrics and Gynaecology .21 Mar 2016.

9. Symonds M, Mike Kagawa, Khadija Malima, Essential Obstetrics and Gynaecology .09 July 2017.

10. Bain, Burton C, McGavigan, Gynaecology Illustrated. 05 Oct 2011.

11. National Institute for Health and Clinical Excellence, NICE Clinical Guideline 154, (Level III), Ectopic pregnancy and miscarriage: diagnosis and initial management in early pregnancy of ectopic pregnancy and miscarriage .January 20 , 2015.

12. Barnhart KT, Fertil Steril, Early pregnancy failure. 98:1061-5. (Level III), 2018.

13. Doubilet $\mathrm{PM}$, Benson $\mathrm{CB}$, Bourne $\mathrm{T}$, Blaivas M, Barnhart KT, Benacerraf BR, et al., N Engl J Med. (Level III), Diagnostic criteria for nonviable pregnancy early in the first trimester. Society of Radiologists in Ultrasound Multispecialty Panel on Early First Trimester Diagnosis of Miscarriage and Exclusion of a Viable Intrauterine Pregnancy.2018; 369 :1443-51.

14. Choobun $\mathrm{T}$, Khanuengkitkong S, Pinjaroen S. (Level II-3), A comparative study of cost of care and duration of management for first-trimester 
abortion with manual vacuum aspiration (MVA) and sharp curettage. 2018; $286: 1161-4$.

15. PetrouS, McIntosh E, Value Health (Level III), Women's preferences for attributes of first-trimester miscarriage management : a stated preference discrete - choice experiment .2015;12:551-9.

16. Rausch M, Lorch S, Chung K, Frederick M, Zhang J, Barnhart K, FertilSteril (Level III), A costeffectiveness analysis of surgical versus medical management of early pregnancy loss .2017; 97 : 355-60 .

17. Colleselli V, Schreiber CA, D'Costa E, Mangesius S, Wildt L, Seeber BE, Arch Gynecol Obstet. (Level II-3), Medical management of early pregnancy failure $(\mathrm{EPF})$ : a retrospective analysis of a combined protocol of mifepristone and misoprostol used in clinical practice .2018; $289: 1341-5$. 